\title{
$D$ meson semileptonic decays from lattice QCD with chiral fermions
}

\section{JLQCD Collaboration: T. Suzuki ${ }^{\star a} \dagger$ Y-G. Cho ${ }^{b}$, H. Fukaya ${ }^{a}$, S. Hashimoto ${ }^{c, d}$,} T. Kaneko ${ }^{c, d}$, J. Noaki ${ }^{c}$

${ }^{a}$ Department of Physics, Osaka University, Toyonaka, Osaka 560-0043 Japan

${ }^{b}$ Department of Physics, Tokyo Institute of Technology, Meguro, Tokyo, 152-8551, Japan

${ }^{c}$ High Energy Accelerator Research Organization (KEK), Tsukuba 305-0801, Japan

${ }^{d}$ School of High Energy Accelerator Science, The Graduate University for Advanced Studies

(Sokendai),Tsukuba 305-0801, Japan

\begin{abstract}
We report on our study of $D$ meson semileptonic decays in QCD with 2+1 flavors of Möbius domain-wall fermions. Gauge ensembles are generated at lattice cut-off around $2.4 \mathrm{GeV}$ and pion masses $300 \mathrm{MeV}$ and above. Relevant form factors are calculated using the Möbius action also for the valence charm quarks.
\end{abstract}

The 33rd International Symposium on Lattice Field Theory

14 -18 July 2015

Kobe International Conference Center, Kobe, Japan*

\footnotetext{
*Speaker.

${ }^{\dagger}$ E-mail:suzuki@het.phys.sci.osaka-u.ac.jp
} 


\section{Introduction}

The semileptonic weak decays of the $D$ meson are used for the determination of the Cabibbo-

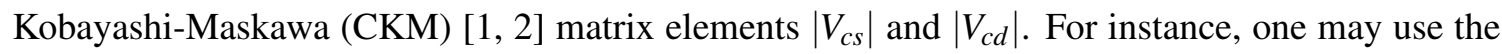
differential decay rate of the process $D \rightarrow \pi \ell v$,

$$
\frac{\Gamma(D \rightarrow \pi \ell v)}{d q^{2}}=\frac{G_{F}^{2}\left|\vec{p}_{\pi}\right|^{3}}{24 \pi^{3}}\left|V_{c d}\right|^{2}\left|f_{+}^{D \rightarrow \pi}\left(q^{2}\right)\right|^{2},
$$

where $q^{2}=\left(p_{D}-p_{\pi}\right)^{2}$ is the momentum transfer, and $\vec{p}_{\pi}$ is the three momentum of the pion $(\pi)$. We have taken the rest frame of the $D$ meson, and neglected the term proportional to the lepton mass squared. The same relation holds for $D \rightarrow K$ by replacing $\pi$ and $\left|V_{c d}\right|$ by $K$ and $\left|V_{c s}\right|$, respectively. In the past decade, several experiments have measured these decay processes precisely and determined its dependence on $q^{2}$ [[1], 田, []].

The task of lattice QCD is to precisely calculate the form factor $f_{+}^{D \rightarrow \pi}\left(q^{2}\right)$. It is defined together with another form factor $f_{-}^{D \rightarrow \pi}\left(q^{2}\right)$ by the $D \rightarrow \pi$ (or $K$ ) hadronic matrix element,

$$
\left\langle\pi\left(p_{\pi}\right)\left|V_{\mu}\right| D\left(p_{D}\right)\right\rangle=\left(p_{D}+p_{\pi}\right)_{\mu} f_{+}^{D \rightarrow \pi}\left(q^{2}\right)+\left(p_{D}-p_{\pi}\right)_{\mu} f_{-}^{D \rightarrow \pi}\left(q^{2}\right),
$$

with $V_{\mu}=\bar{c} \gamma_{\mu} d$. By combining the matrix elements with various momenta $p_{D}$ and $p_{\pi}\left(p_{K}\right)$, one can extract the form factors.

In this talk, we report on our preliminary results for the $D \rightarrow \pi$ and $D \rightarrow K$ form factors. We employ the Möbius domain-wall fermion for both heavy and light quarks. The residual violation of chiral symmetry is small, i.e. the residual mass is of $\mathscr{O}(1 \mathrm{MeV})$.

\section{Method}

To extract the hadronic matrix elements, we define

$$
\begin{aligned}
A_{\mu}^{D \rightarrow \pi}\left(\mathbf{p}_{D}, \mathbf{p}_{\pi}\right) & =\frac{C_{\mu}^{D \rightarrow \pi}\left(\Delta t, \Delta t^{\prime} ; \mathbf{p}_{D}, \mathbf{p}_{\pi}\right)}{\exp \left(-E_{D} \Delta t-E_{\pi} \Delta t^{\prime}\right)} \\
B^{D(\pi)}\left(\mathbf{p}_{D(\pi)}\right) & =\frac{C^{D(\pi)}\left(\Delta t ; \mathbf{p}_{D(\pi)}\right)}{\exp \left(-E_{D(\pi)} \Delta t\right)}
\end{aligned}
$$

with $E_{D(\pi)}=\sqrt{m_{D(\pi)}^{2}+\mathbf{p}_{D(\pi)}^{2}}$ the energy of $D$ or $\pi$ meson, for which we assume the dispersion relation. The two- and three- point functions are calculated as

$$
\begin{aligned}
C_{\mu}^{D \rightarrow \pi}\left(\Delta t, \Delta t^{\prime} ; \mathbf{p}_{D}, \mathbf{p}_{\pi}\right) \equiv \frac{1}{V} \sum_{\mathbf{x}, t} \sum_{\mathbf{x}^{\prime}, \mathbf{x}^{\prime \prime}}\left\langle\mathscr{O}_{\pi}\left(\mathbf{x}^{\prime \prime}, t+\Delta t+\Delta t^{\prime}\right) V_{\mu}\left(\mathbf{x}^{\prime}, t+\Delta t\right) \mathscr{O}_{D}^{\dagger}(\mathbf{x}, t)\right\rangle \\
\times e^{-i \mathbf{p}_{D}\left(\mathbf{x}^{\prime \prime}-\mathbf{x}^{\prime}\right)} e^{-i \mathbf{p}_{\pi}\left(\mathbf{x}^{\prime}-\mathbf{x}\right)}, \\
C^{D(\pi)}\left(\Delta t ; \mathbf{p}_{D(\pi)}\right) \equiv \frac{1}{V} \sum_{\mathbf{x}, t} \sum_{\mathbf{x}^{\prime}}\left\langle\mathscr{O}_{D(\pi)}\left(\mathbf{x}^{\prime}, t+\Delta t\right) \mathscr{O}_{D(\pi)}^{\dagger}(\mathbf{x}, t)\right\rangle e^{-i \mathbf{p}_{D(\pi)}\left(\mathbf{x}^{\prime}-\mathbf{x}\right)}
\end{aligned}
$$

with the meson interpolating operator $\mathscr{O}(\mathbf{x}, t)=\sum_{\mathbf{x}^{\prime}} \phi\left(\mathbf{x}^{\prime}\right) \bar{q}\left(\mathbf{x}+\mathbf{x}^{\prime}, t\right) \gamma_{5} q(\mathbf{x}, t)$ where $\phi\left(\mathbf{x}^{\prime}\right)$ represents the Gaussian smearing operator: $(1-(\alpha / N) \Delta)^{N}$ with the Laplacian $\Delta, \alpha=5.0$ and $N=50$. 
Table 1: Simulation Parameters

\begin{tabular}{cccccc}
\hline$\beta$ & $a^{-1}$ & Volume $\left(L^{3} \times T\right)$ & $a m_{s}$ & $a m_{u d}$ & \# of source points \\
\hline \hline & & & & 0.007 & 8 \\
4.17 & $2.453(4) \mathrm{GeV}$ & $32^{3} \times 64$ & 0.04 & 0.012 & 5 \\
& & & & 0.019 & 2 \\
\hline
\end{tabular}

From the asymptotic behavior of $A_{\mu}^{D \rightarrow \pi}\left(\mathbf{p}_{D}, \mathbf{p}_{\pi}\right)$ and $B^{D(\pi)}\left(\mathbf{p}_{D(\pi)}\right)$ for large $\Delta t$ and $\Delta t^{\prime}$, we can extract the desired hadronic matrix elements as

$$
R_{\mu}^{D \rightarrow \pi}\left(\mathbf{p}_{D}, \mathbf{p}_{\pi}\right) \equiv 2 Z_{V} \sqrt{E_{D} E_{\pi}} \sqrt{\frac{\left|A_{\mu}^{D \rightarrow \pi}\left(\mathbf{p}_{D}, \mathbf{p}_{\pi}\right)\right|^{2}}{B^{D}\left(\mathbf{p}_{D}\right) B^{\pi}\left(\mathbf{p}_{\pi}\right)}} \stackrel{\text { large } \Delta t, \Delta t^{\prime}}{\longrightarrow}\left\langle\pi\left|V_{\mu}\right| D\right\rangle,
$$

with the renormalization factor of the vector current $Z_{V}$, which is calculated non-perturbatively in [四] . For $q^{2}<q_{\max }^{2}=\left(m_{D}-m_{\pi}\right)^{2}$, the form factors can be computed from these $R$ 's as

$$
\begin{aligned}
& f_{+}^{D \rightarrow \pi}\left(q^{2}\right)=\frac{\left(E_{D}-E_{\pi}\right) R_{k}^{D \rightarrow \pi}\left(\mathbf{p}_{D}, \mathbf{p}_{\pi}\right)-\left(p_{D}-p_{\pi}\right)^{k} R_{4}^{D \rightarrow \pi}\left(\mathbf{p}_{D}, \mathbf{p}_{\pi}\right)}{2 E_{D} p_{\pi}^{k}-2 E_{\pi} p_{D}^{k}}, \\
& f_{-}^{D \rightarrow \pi}\left(q^{2}\right)=\frac{\left(E_{D}+E_{\pi}\right) R_{k}^{D \rightarrow \pi}\left(\mathbf{p}_{D}, \mathbf{p}_{\pi}\right)-\left(p_{D}+p_{\pi}\right)^{k} R_{4}^{D \rightarrow \pi}\left(\mathbf{p}_{D}, \mathbf{p}_{\pi}\right)}{2 E_{\pi} p_{D}^{k}-2 E_{D} p_{\pi}^{k}}, \\
& f_{0}^{D \rightarrow \pi}\left(q^{2}\right)=f_{+}^{D \rightarrow \pi}\left(q^{2}\right)+f_{-}^{D \rightarrow \pi}\left(q^{2}\right) \frac{q^{2}}{\left(m_{D}^{2}-m_{\pi}^{2}\right)},
\end{aligned}
$$

where $k$ denotes the $k$-th spatial component, and $f_{0}\left(q^{2}\right)$ is the scalar form factor. At $q^{2}=q_{\max }^{2}$, one can only access to $f_{0}\left(q_{\max }^{2}\right)$, which can be obtained by

$$
\frac{R_{4}^{D \rightarrow \pi}(\mathbf{0}, \mathbf{0})}{m_{D}+m_{\pi}} \cdot \stackrel{\text { large } \Delta t, \Delta t^{\prime}}{\longrightarrow} f_{0}^{D \rightarrow \pi}\left(q_{\max }^{2}\right)
$$

Note that all the relations in this section hold for the $D \rightarrow K$ case by replacing $\pi$ by $K$.

\section{Lattice set up}

We use the 2+1-flavor gauge ensembles generated with the Symanzik gauge action and the Möbius domain-wall fermion action with three times stout smearing of the link variables. In this work, we use the lattice of size $L^{3} \times T\left(\times L_{s}\right)=32^{3} \times 64(\times 12)$ at $\beta=4.17$, of which the lattice cut-off is estimated to be $2.453(4) \mathrm{GeV}$ using the Wilson flow.

The parameters are summarized in Table $\mathrm{W}$. We use three values of the up and down quark masses, $a m_{u d}=0.007,0.012$ and 0.019. The strange quark mass is set to be $a m_{s}=0.04$ for all $m_{u d}$. The corresponding pion mass is 308,399 , and $498 \mathrm{MeV}$, respectively. On each ensemble, 100 configurations are sampled from 10,000 HMC trajectories and 2-8 different source points are taken to improve statistics. We estimate the error by the jackknife method with bin-size 4 .

The same ensembles are also used for a calculation of the charmed meson decay constants $f_{D}$ and $f_{D_{s}}[$ [ $]$. We found that the discretization effect is not significant for these quantified by comparing the results with those on finer lattices at $\beta=4.35$ and 4.47 . 

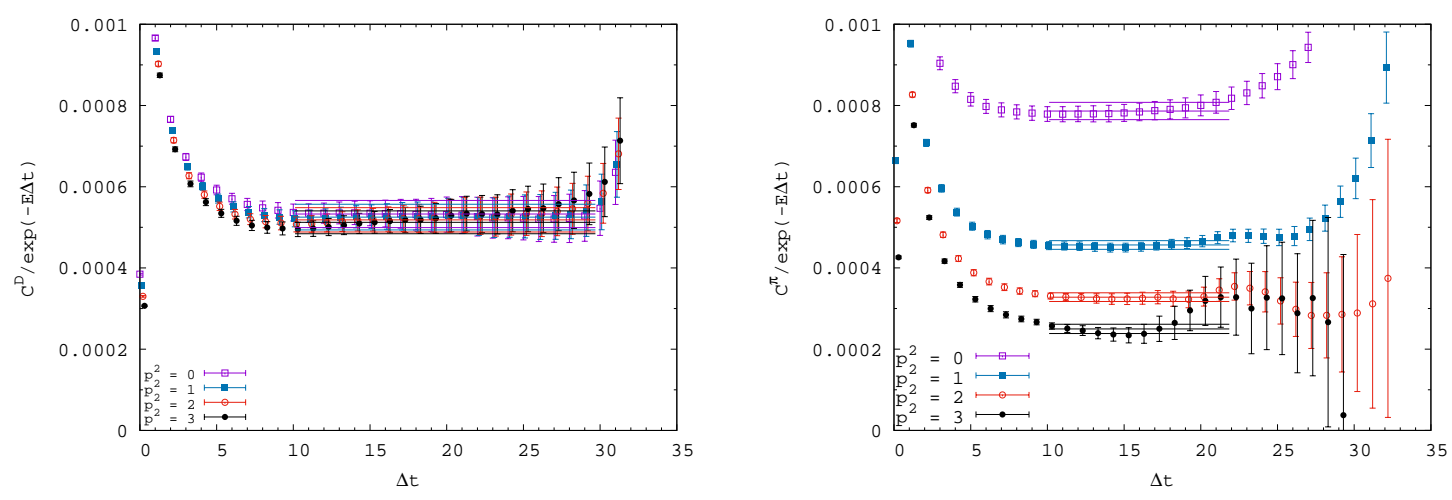

Figure 1: $B^{D}\left(\mathbf{p}_{D}\right)$ (left) and $B^{\pi}\left(\mathbf{p}_{\pi}\right)$ (right) mesons. The horizontal axis is the temporal distance of two-point function. Data with four different momenta are shown. The corresponding pion mass is $399 \mathrm{MeV}$. The factor $B^{D / \pi}\left(\mathbf{p}_{D / \pi}\right)$ is extracted from the plateaus, as shown by the bands.
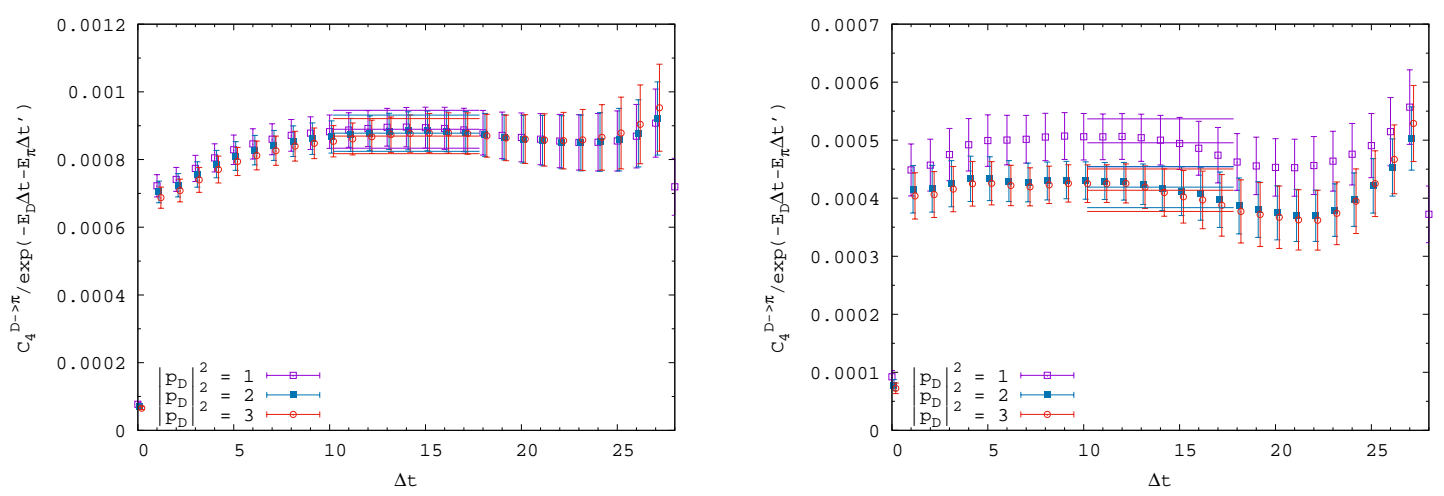

Figure 2: $A_{\mu}^{D \rightarrow \pi}\left(\mathbf{p}_{D}, \mathbf{p}_{\pi}\right)$ with $\mathbf{p}_{\pi}=\mathbf{0}$ (left) or $\mathbf{p}_{\pi} \neq \mathbf{0}$ (right). Data for three different momenta $\mathbf{p}_{D}$ of $\mathrm{D}$ meson are plotted. The horizontal axis indicates the temporal position of the current operator. The corresponding pion mass is $399 \mathrm{MeV}$. Source and sink operators are put at $t=0$ and $t+\Delta t+\Delta t^{\prime}=28$, respectively.

\section{Numerical results}

First, we compute the r.h.s. of Eqs. (‥ 1 ) and (2.2) to extract the factors $A_{\mu}^{D \rightarrow \pi}\left(\mathbf{p}_{D}, \mathbf{p}_{\pi}\right)$ and $B^{D / \pi}\left(\mathbf{p}_{D / \pi}\right)$ at large separations. Typical results are shown in Figs. \and $\square$. In Fig. \, the horizontal axis $\Delta t$ denotes the temporal position of current operator, and we set the source and sink operators at $t=0$ and $t+\Delta t+\Delta t^{\prime}=28$, respectively. As already mentioned, the simple dispersion relation for the meson energy is assumed. We observe reasonable plateau on every ensemble and the factors $A_{\mu}^{D \rightarrow \pi}\left(\mathbf{p}_{D}, \mathbf{p}_{\pi}\right)$ and $B^{D / \pi}\left(\mathbf{p}_{D / \pi}\right)$ are determined by a constant fit, which is shown in the plots by a horizontal line.

Next, we extract the form factors through Eqs. (12.6)-(12.8). Our result for the form factor $f_{+}\left(q^{2}\right)$ at the three pion masses are shown in Fig. B as a function of the momentum transfer $q^{2}$. The solid curve is a single pole fit of the experimental data by CLEO-c [园] (with an input for $\left|V_{c d(s)}\right|$ from PDG [U]]), which shows a reasonable agreement with our data suggesting a mild $m_{\pi}$ dependence 

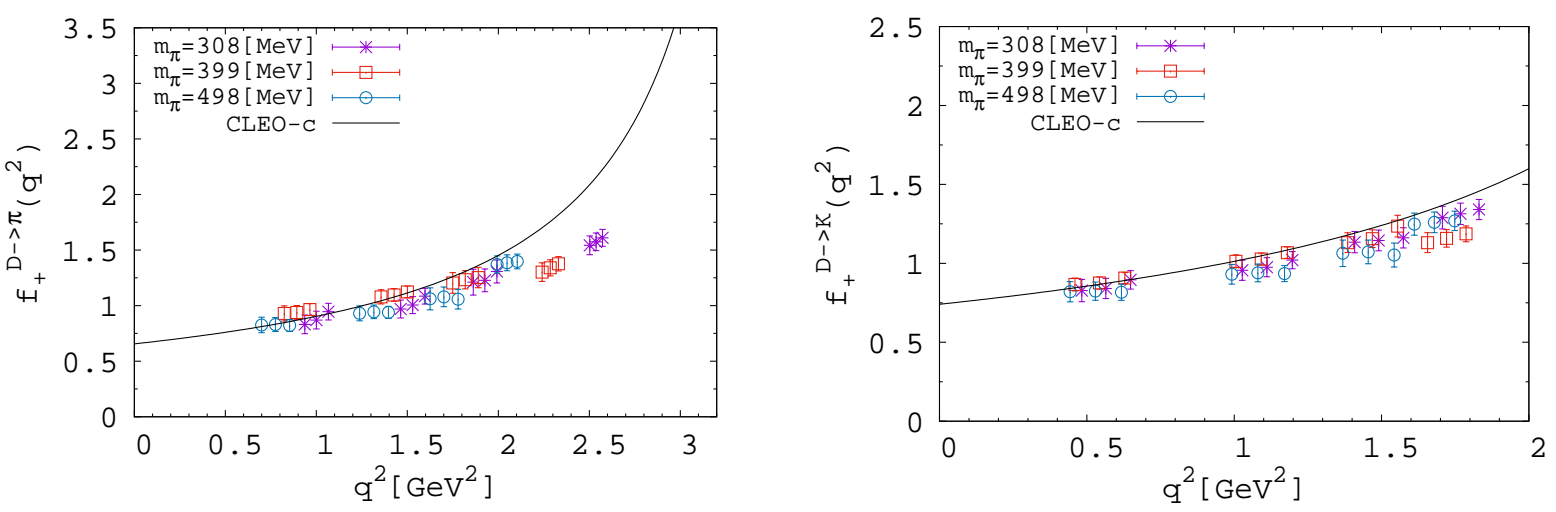

Figure 3: $q^{2}$ dependence of $f_{+}\left(q^{2}\right)$ of $D \rightarrow \pi$ (left) and $D \rightarrow K$ (right). Different colors represent different pion masses. The solid curve shows a single pole fit of the experimental data by CLEO-c [B]].

of $f_{+}\left(q^{2}\right)$. The CKM matrix elements $\left|V_{c d}\right|$ and $\left|V_{c s}\right|$ are conventionally determined from the form factor at zero momentum transfer $f_{+}(0)$. In this work, we determine $f_{+}(0)$ by extrapolating the data with fit functions based on the Vector Meson Dominance (VMD) hypothesis:

$$
f_{+}^{f i t}\left(q^{2}\right)=\frac{f_{+}(0)}{1-q^{2} / m_{V}^{2}} \times(\text { Polynomial }) .
$$

The vector meson pole mass $m_{V}$ is the $m_{D^{*}}$ and $m_{D_{s}^{*}}$ in the case of $D \rightarrow \pi$ and $D \rightarrow K$, respectively. We use the value of $m_{V}$ measured at simulated pion masses. We use three types of "Polynomial" in Eq. (4. (ل) : $1,1+a q^{2}$, and $1+a q^{2}+b\left(q^{2}\right)^{2}$. Namely, $f_{+}(0), a$ and $b$ are the unknown fit parameters in our analysis. The results are shown in Fig. 团. The value of $\chi^{2} /$ d.o.f. is $1.9,0.25$ and 0.045 for $1,1+a q^{2}$, and $1+a q^{2}+b\left(q^{2}\right)^{2}$, respectively (for $D \rightarrow \pi$ at $m_{\pi}=399 \mathrm{MeV}$ ). As one can see from Fig. B]- 3 , the simple VMD form fails to describe the data near $q_{\max }^{2}$. Since the results for the fit parameter $b$ are mostly consistent with zero, we use the fit function $\mathrm{VMD} *\left(1+a q^{2}\right)$ in the following analysis.

Our simulated pion masses are heavier than the physical point, and we need to perform a chiral extrapolation of $f_{+}(0)$. As seen in Fig. 15, it turned out that the $m_{\pi}$ dependence of $f_{+}(0)$ is mild. Here the results with the fit function $\mathrm{VMD} *\left(1+a q^{2}\right)$ are shown. We therefore determine $f_{+}(0)$ at the physical pion mass by a simple constant fit in this preliminary analysis. Within the error-bars, the results are consistent with the current world average by FLAG [8] (or [Q, [0]), which is shown by the black points.

\section{Summary}

In this work, we compute the form factors for the $D$ meson semileptonic decays by lattice QCD with chiral fermions for both heavy and light quarks. Our preliminary results for the form factors at $q^{2}=0$ at the physical pion mass are

$$
f_{+ \text {phys }}^{D \rightarrow \pi}(0)=0.720(45)_{\text {stat }}(41)_{\text {sys }}, \quad f_{+ \text {phys }}^{D \rightarrow K}(0)=0.757(29)_{\text {stat }}(02)_{\text {sys }},
$$



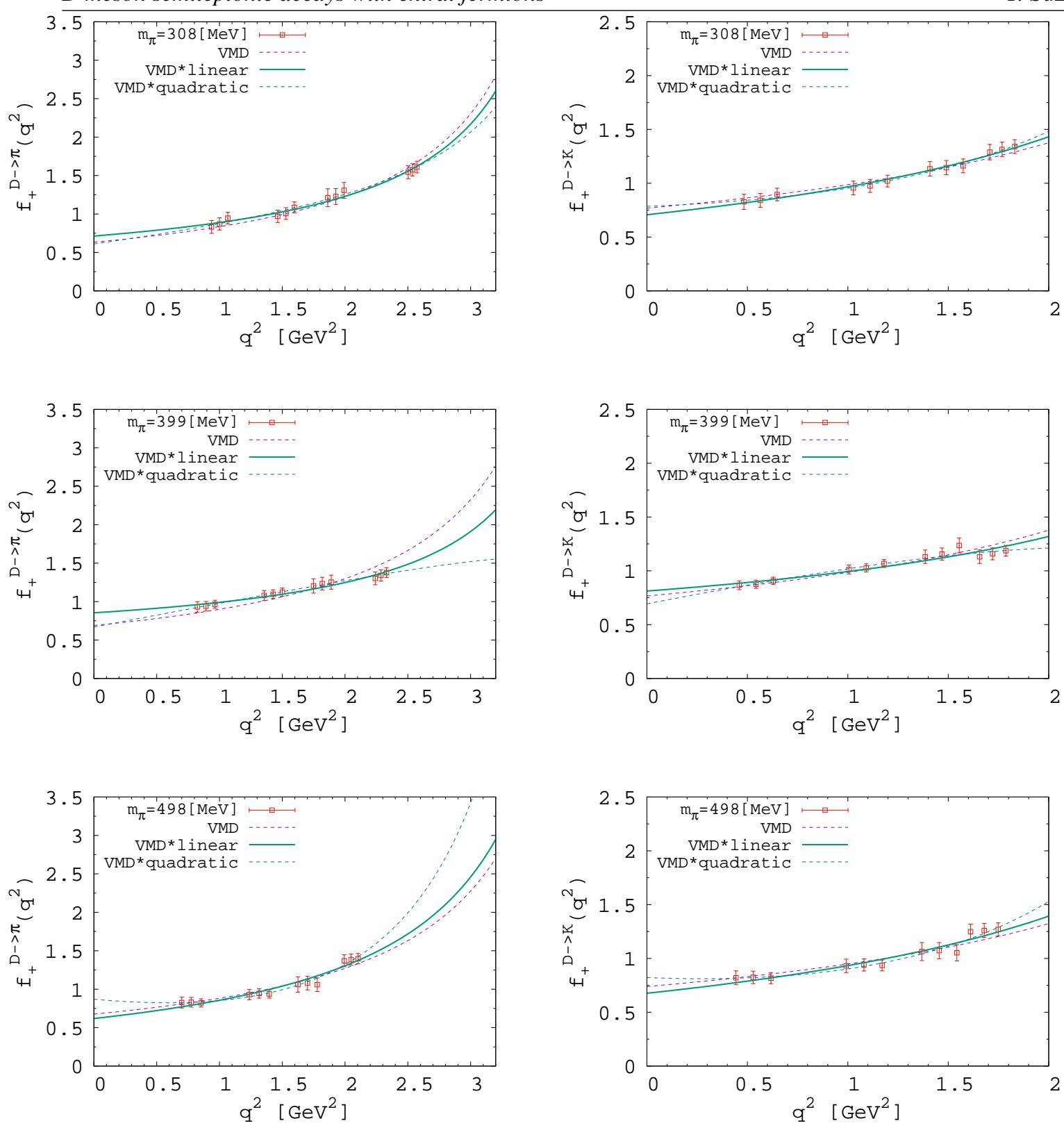

Figure 4: Our fit results to $f_{+}\left(q^{2}\right)$ of $D \rightarrow \pi$ (left) and $D \rightarrow K$ (right). The simulated pion masses are 308, 399 and $498 \mathrm{MeV}$. Curves represent the fits motivated by the VMD hypothesis. See the text for more details.

where the first error is statistical. The second one is the systematic error, which is estimated from the difference of the results with various fit functions. Combined with the CLEO-c [B] results, $f_{+}^{D \rightarrow \pi}(0)\left|V_{c d}\right|=0.148$ and $f_{+}^{D \rightarrow K}(0)\left|V_{c s}\right|=0.721$, our preliminary results for the CKM matrix elements are $\left|V_{c d}\right|=0.205(12)_{s t a t}(11)_{s y s},\left|V_{c s}\right|=0.952(36)_{s t a t}(02)_{\text {sys. }}$. These are consistent with $\left|V_{c d}\right|=0.22522(61),\left|V_{c s}\right|=0.97343(15)$ in PDG [U]]. Although we have used the parametrization of the form factors based on VMD, another parametrization (for example the z-expansion [ए2]) would be helpful to reduce the systematic error. 

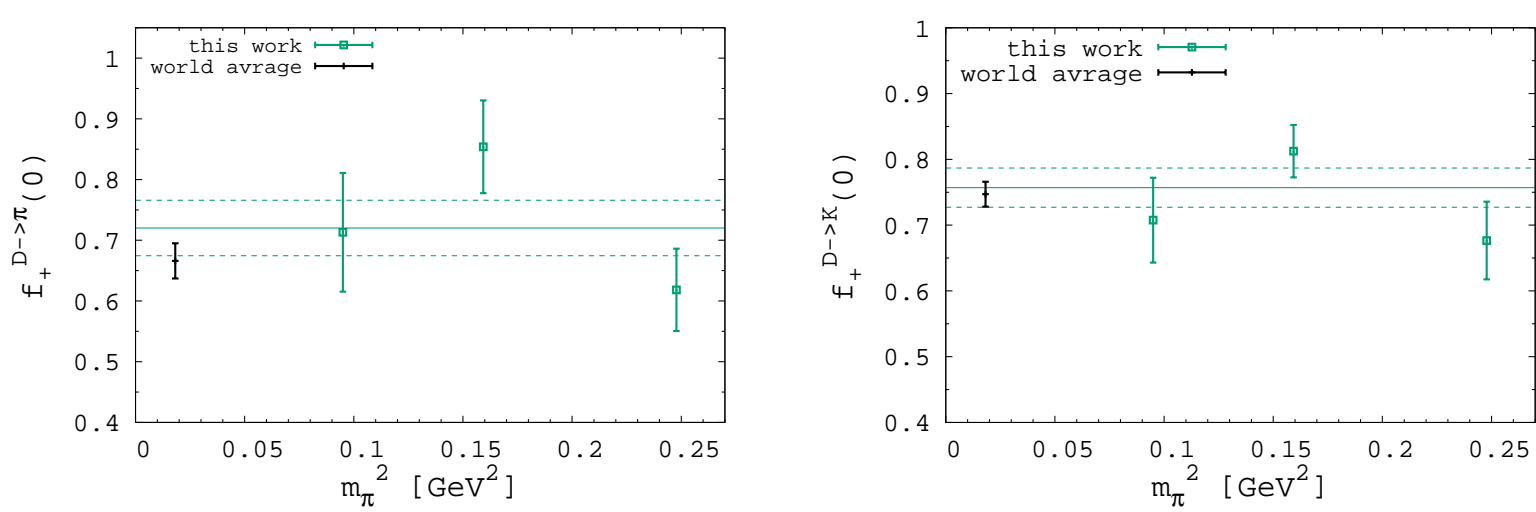

Figure 5: $f_{+}(0)$ of $D \rightarrow \pi$ (left) and $D \rightarrow K$ (right). The black points are the values by FLAG [8]

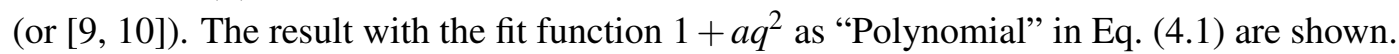

\section{Acknowledgements}

Numerical simulations are performed on IBM System Blue Gene Solution at the High Energy Accelerator Research Organization (KEK) under a support of its Large Scale Simulation Program (No. 13/14-4, 14/15-10). This work is supported in part by the Grant-in-Aid of the Japanese Ministry of Education (No. 26400259, 15K05065). We sincerely thank other members of JLQCD collaboration for fruitful discussions.

\section{References}

[1] N. Cabibbo, Phys. Rev. Lett. 10, 531 (1963).

[2] M. Kobayashi and T. Maskawa, Prog. Theor. Phys. 49, 652 (1973).

[3] D. Besson et al. [CLEO Collaboration], Phys. Rev. D 80, 032005 (2009) [arXiv:0906.2983 [hep-ex]].

[4] B. Aubert et al. [BaBar Collaboration], Phys. Rev. D 76, 052005 (2007) [arXiv:0704.0020 [hep-ex]].

[5] L. Widhalm et al. [Belle Collaboration], Phys. Rev. Lett. 97, 061804 (2006) [hep-ex/0604049].

[6] M. Tomii et al. [JLQCD Collaboration], arXiv:1511.09170 [hep-lat].

[7] B. Fahy, G. Cossu, S. Hashimoto, T. Kaneko, J. Noaki, and M. Tomii [JLQCD Collaboration], PoS (LATTICE 2015) 074.

[8] S. Aoki et al., Eur. Phys. J. C 74, 2890 (2014) [arXiv:1310.8555 [hep-lat]].

[9] H. Na, C. T. H. Davies, E. Follana, J. Koponen, G. P. Lepage and J. Shigemitsu, Phys. Rev. D 84, 114505 (2011) doi:10.1103/PhysRevD.84.114505 [arXiv:1109.1501 [hep-lat]].

[10] H. Na, C. T. H. Davies, E. Follana, G. P. Lepage and J. Shigemitsu, Phys. Rev. D 82, 114506 (2010) doi:10.1103/PhysRevD.82.114506 [arXiv:1008.4562 [hep-lat]].

[11] K. A. Olive et al. [Particle Data Group Collaboration], Chin. Phys. C 38, 090001 (2014).

[12] C. Bourrely, I. Caprini and L. Lellouch, Phys. Rev. D 79, 013008 (2009) [Phys. Rev. D 82, 099902 (2010)] [arXiv:0807.2722 [hep-ph]]. 